\title{
ASSESSMENT OF PLATELET COUNT AND DOPPLER ULTRASOUND ON HEPATIC AND SPLENIC VENOUSSYSTEM AS NON-INVASIVE PREDICTORS OF GASTRIC VARICES IN PATIENTS WITH LIVER CIRRHOSIS
}

\author{
By \\ Osama Sayed Ahmed Ahmed Ghalwash, Gamal Mohammad \\ Mohammad Soliman, Galal Abd El-Hameed Abou-Farrag and \\ Mohammad Abolnaga Mohammad* \\ Departments of Hepatogastroenterology\& infectious diseases and Radio diagnosis*, \\ Faculty of Medicine, Al-Azhar University, Cairo, Egypt \\ Corresponding author: Osama Sayed Ahmed Ahmed Ghalwash \\ E-mail: osamaghalwash26490@gmail.com
}

\begin{abstract}
Background: Gastric varices are probably associated with thrombocytopenia. However, the prevalence and severity of thrombocytopenia are unknown in this clinical setting.

Objective: To assess platelet count and doppler ultrasound on hepatic and splenic venous system as noninvasive predictors of gastric varices in patients with liver cirrhosis with no history of previous endoscopic or surgical intervention for portal hypertension.

Patients and Methods: An observational case control study was accomplished at Al-Azhar University Hospitals, Hepatogastroenterology and infectious diseases Departments, at Cairo from September 2018 to December 2019. One hundred and twenty patients with liver cirrhosis without past history of previous endoscopic or surgical intervention for portal hypertension were selected, and divided into three equal main groups:Group A: Cirrhotic patients without varices, Group B: Cirrhotic patients with esophageal varices only, and Group C: Cirrhotic patients with gastric varices, which divided into two subgroups: Group Ca:Six patients with isolated gastric varices and Group Cb:Thirty four patients with gastroesophageal varices. All patients were subjected to upper gastrointestinal endoscopy,complete blood picture (CBC) and abdominal Doppler ultrasonography study on hepatic and splenic venous systems.

Results: Group $\mathrm{Cb}$ recorded the highest values of splenic size $(\mathrm{P}<0.001)$, ascites $(\mathrm{p}=0.006)$, portal vein diameter $(\mathrm{P}<0.001)$ and collaterals $(\mathrm{P}<0.001)$ compared to other groups. Group $\mathrm{Ca}$ recorded the lowest values of the mean portal vein flow velocity $(\mathrm{MPVV})(\mathrm{p}<0.001)$, the mean splenic vein flow velocity(M.SVV) $(\mathrm{P}=0.026)$, and recorded the highest values of portal vein congestion index (P.CI) $(\mathrm{P}<0.001)$, portal vein thrombosis $(\mathrm{PVT})(\mathrm{P}<0.001)$, the mean values of the splenic vein diameter $(\mathrm{P}<0.001)$, cross sectional area of splenic vein $(\mathrm{p}<0.001)$, splenic vein congestion index(S.CI) $(\mathrm{p}<0.001)$, as well as abnormal blood direction in splenic vein $(\mathrm{P}=0.019)$ compared to other groups. Also, portal vein cross sectional area and the ChildTurcotte-Pugh score class "C" showed statistically significant difference $(p<0.001)$ between group A which recorded the lowest value compared to group B which recorded the highest value. Also, platelet count recorded no statistically significant difference between the four groups.
\end{abstract}

Conclusion: Doppler ultrasound can be an easy, cheap and safe predictor of gastric varices, while platelet count has no any significance in predicting of gastric varices.

Keywords: Cirrhosis, platelet count, Doppler ultrasound, hepatic venous, splenic venous, gastric varices. 


\section{INTRODUCTION}

Chronic liver diseases impose a major burden in health systems. Although of different etiologies, they share common end stage namely liver cirrhosis with portal hypertension (PHT) (Sauerbruch and Trebicka, 2014). The most common cause of PHT is cirrhosis while vascular resistance and blood flow are the two important factors in its development (Jesus, 2017).

Portal hypertension (PHT) can cause a variety of pathologic changes along the entire gastrointestinal tract from the esophagus to the anus which manifest as varices, gastropathy, and enteropathy ( $D i$ Giorgio and D'Antiga, 2019).

Gastric varices $(\mathrm{GV})$ are less prevalent than esophageal varices (EV) occurring in approximately $20 \%$ of patients with PHT with a reported incidence of bleeding of $10-30 \%$ of all variceal hemorrhages with a higher bleeding incidence for fundal varices. They are developed due to spontaneous portosystemic collaterals commonly between the splenic and gastric veins. Thus, gastric varices are commonly classified based on their relationship with esophageal varices as well as their location in the stomach (Zeng et al., 2017).

Patients with compensated cirrhosis and small varices with no high-risk stigmata may be considered for endoscopic variceal surveillance every $1-$ 2 years to evaluate progression, in patients with advanced liver disease or medium or large varices, primary prophylaxis should be implemented (Garcia-Tsao et al., 2017).
Various noninvasive hematological, biochemical and ultra-sonographic predictors have been suggested which include splenic size, portal vein diameter, serum albumin levels and platelet count; all of these parameters are non-invasive and easy to perform (DeFranchis and Faculty, 2015).

Gastric varices primarily occur in cirrhotic patients with portal hypertension and splenomegaly and thus are probably associated with thrombocytopenia. However, the prevalence and severity of thrombocytopenia are unknown in this clinical setting (Wael et al., 2014).

Doppler ultrasonography (US) imaging is considered the first-line imaging technique in patients with cirrhosis. Portal vein diameter, portal blood velocity and congestion index, spleenic size, flow pattern in the hepatic veins, and the presence of abdominal portosystemic collaterals are all US parameters previously thought to be associated with prognostic significance (Rye et al., 2012).

This study aimed to assess platelet count and doppler ultrasound on hepatic and splenic venous system as noninvasive predictors of gastric varices in patients with liver cirrhosis with no history of previous endoscopic or surgical intervention for portal hypertension.

\section{PATIENTS AND METHODS}

An observational case control study accomplished at Al-Azhar university hospitals, Hepatogastroenterology and infectious diseases departments at Cairo according to the ethical board of Al-Azhar University from September 2018 to December 2019 where 120 patients aged more than 18 years old with liver cirrhosis 
without past history of previous endoscopic or surgical intervention for portal hypertension were selected. Study information sheet was provided to patients and informed consents were written by patients who agreed to participate in this study.

Patients were divided into three equal main groups: Group A: Forty cirrhotic patients without varices, Group B: Forty cirrhotic patients with esophageal varices only and Group C: Forty cirrhotic patients with gastric varices, which divided into two subgroups, Group $\mathrm{Ca}$ with isolated gastric varices and Group $\mathrm{Cb}$ with gastroesophageal varices.

We excluded patients with previous endoscopic or surgical intervention for portal hypertension, patients with associated advanced co-morbidity (as advanced cardiac disease, renal disease, cancer liver ...etc) and patients refused to sign an informed consent.

All participants were subjected to full history taking and clinical examination including manifestations of chronic liver disease (such as jaundice, flapping tremors, lower limb edema, organomegaly, ascites), routine laboratory investigations including complete blood picture (CBC), Liver profile (aminotransferases (ALT\&AST), serum albumin, total\& direct bilirubin, prothrombin time and INR) andrenal function tests (serum creatinine and blood urea),Child-Turcotte-Pugh scoreclassification, abdominal ultrasonography and doppler study with emphasis on: liver size (classified as shrunken $<11 \mathrm{~cm}$, average $11-15 \mathrm{~cm}$ or enlarged $>15 \mathrm{~cm}$ ), criteria suggestive of chronic liver disease and cirrhosis, presence of periportal thickening, splenic bi-polar diameter (normal up to $12-13 \mathrm{~cm}$ ), ascites (reported as mild, moderate or marked ascites if present), portal vein indices including: portal vein diameter (PVD) and patency or thrombosis (PVT), cross sectional area (A), mean portal vein flow velocity (MPVV), congestion index of the portal vein $(\mathrm{P} . \mathrm{CI}=\mathrm{A}(\mathrm{cm} 2) /$ mean PVV $(\mathrm{cm} / \mathrm{sec})$ anddirection of flow (hepatopetal, bidirectional orhepatofugal), splenic vein indices including: splenic vein diameter $(\mathrm{mm})$ and patency, cross sectional area (A), mean splenic vein flow velocity ( MSVV), congestion index of the splenic vein $(\mathrm{S} . \mathrm{CI}=\mathrm{A}(\mathrm{cm} 2) /$ mean $\mathrm{SVV}(\mathrm{cm} / \mathrm{sec})$ and direction of flow (hepatopetal, bidirectional, hepatofugal), hepatic vein patency and dilation and presence of portosystemic collaterals, also upper gastrointestinal endoscopy was done to all participants to evaluate the presence of esophageal varices and its grade and the presence and type of gastric varices based upon Sarin classification.

Statistical analysis: Data were analyzed using Statistical Package for the Social Sciences (SPSS) version 21.Quantitative data were expressed as mean \pm standard deviation (SD), Also qualitative data were expressed as frequency and percentage.

\section{The following tests were done:}

- Chi-Square test ( $\chi^{2}$ value): It is used to compare between two groups or more regarding one qualitative variable.

- Fisher's exact test: It is used to compare between two groups regarding one qualitative variable in a $2 \times 2$ contingency table when the expected count of any of the cells less than 5. 
- One-way ANOVA (F) test was used to compare more than two groups for continuous variables.

- Pearson correlation.

- Probability (P-value) P-value<0.05 was considered significant.
- Kruskal - Wallis test, Pairwise comparison between each to groups was done using Post Hoc test (Dunn, s for multiple comparison test).

\section{RESULTS}

In this study, the age range of participants was from 18 to 72 years with a mean age \pm SD of $(53.63 \pm 9.05$, $56.08 \pm 7.72,54.79 \pm 10.71$ and $54.79 \pm 10.71$ year) for group A, group B, group Ca and group $\mathrm{Cb}$ respectively, showing no statistically significant difference between the four groups $(p=0.55)$.andthe number of males within group $\mathrm{A}$, group $\mathrm{B}$, group $\mathrm{Ca}$ and group $\mathrm{Cb}$ were $(24(60 \%), 32$ (80\%), 4 (66.67\%), and $25(73.53 \%))$ respectively, showing no statistically significant difference between the four groups $(\mathrm{p}=0.25)$ (Table $\mathbf{1})$.

Table (1): Comparison between the studied groups regarding age and sex

\begin{tabular}{|c|c|c|c|c|c|c|c|c|c|}
\hline $\begin{array}{l}\text { Groups } \\
\text { Parameters }\end{array}$ & \multicolumn{2}{|c|}{$\begin{array}{c}\text { Group A } \\
(n=40)\end{array}$} & \multicolumn{2}{|c|}{$\begin{array}{c}\text { Group B } \\
(n=40)\end{array}$} & \multicolumn{2}{|c|}{$\begin{array}{c}\text { Group Ca } \\
(n=6)\end{array}$} & \multicolumn{2}{|c|}{$\begin{array}{c}\text { Group Cb } \\
(n=34)\end{array}$} & $\begin{array}{c}P \\
\text { value }\end{array}$ \\
\hline \multicolumn{10}{|l|}{ Age (years) } \\
\hline Mean \pm SD & \multicolumn{2}{|c|}{$53.63 \pm 9.05$} & \multicolumn{2}{|c|}{$56.08 \pm 7.72$} & \multicolumn{2}{|c|}{$57.83 \pm 5.31$} & \multicolumn{2}{|c|}{$54.79 \pm 10.71$} & \multirow{3}{*}{0.55} \\
\hline Range & \multicolumn{2}{|c|}{$30-71$} & \multicolumn{2}{|c|}{$38-72$} & \multicolumn{2}{|c|}{$51-65$} & \multicolumn{2}{|c|}{ 18-68 } & \\
\hline & No & $\%$ & No & $\%$ & No & $\%$ & No & $\%$ & \\
\hline Sex & & & & & & & & & \\
\hline Males & 24 & 60.0 & 32 & 80.0 & 4 & 66.67 & 25 & 73.53 & 0.25 \\
\hline Females & 16 & 40.0 & 8 & 20.0 & 2 & 33.33 & 9 & 26.47 & \\
\hline Total & 40 & 100.0 & 40 & 100.0 & 6 & 100.0 & 34 & 100.0 & \\
\hline
\end{tabular}

$\chi 2$ : chi square, SD: standard deviation, Group A: cirrhotic patients without varices, Group B: cirrhotic patients with esophageal varices only, Group $\mathrm{Ca}$ : cirrhotic patients with isolated gastric varicesGroup $\mathrm{Cb}$ : cirrhotic patients with gastroesophageal varices.

The mean values of white blood cell counts (WBCs) were $7170 \pm 2634,11.90 \pm 1.69,10.39 \pm 2.12$ and $10.99 \pm 1.98$ in group A, group B, group Ca and group $\mathrm{Cb}$ respectively with statistically significant difference between the four groups $(\mathrm{P}<0.001)$. The mean values of hemoglobin levels (HB) and Platelet counts in group A, group B, group $\mathrm{Ca}$ and group $\mathrm{Cb}$ showed no statistically significant difference between the four groups (Table 2). 
Table (2): Comparison between the studied groups regarding CBC results

\begin{tabular}{|c|c|c|c|c|c|}
\hline Parameters & $\begin{array}{c}\text { Group A } \\
(\mathbf{n}=\mathbf{4 0})\end{array}$ & $\begin{array}{c}\text { Group B } \\
(\mathbf{n}=\mathbf{4 0})\end{array}$ & $\begin{array}{l}\text { Group Ca } \\
(\mathbf{n}=6)\end{array}$ & $\begin{array}{c}\text { Group Cb } \\
(\mathbf{n}=\mathbf{3 4})\end{array}$ & $\begin{array}{c}\text { P- } \\
\text { value }\end{array}$ \\
\hline \multicolumn{6}{|l|}{$\mathbf{H b}(\mathrm{g} / \mathrm{dL})$} \\
\hline Mean \pm SD & $11.59 \pm 1.93$ & $11.90 \pm 1.69$ & $10.39 \pm 2.12$ & $10.99 \pm 1.98$ & \multirow{2}{*}{0.098} \\
\hline Range & $7.90-15.48$ & $8.00-15.50$ & $8.30-14.30$ & $7.90-15.70$ & \\
\hline \multicolumn{6}{|l|}{ WBCs $\left(\right.$ cells $\left./ \mathrm{mm}^{3}\right)$} \\
\hline Mean \pm SD & $7170 \pm 264$ & $4904 \pm 2063$ & $7214 \pm 2286$ & $5542 \pm 2889$ & \multirow{2}{*}{$<0.001$} \\
\hline Range & $2890-12400$ & $1800-10400$ & $3900-10200$ & $2100-13500$ & \\
\hline $\begin{array}{l}\text { Significance } \\
\text { between Groups }\end{array}$ & \multicolumn{4}{|c|}{$\begin{array}{c}\mathrm{p}_{1}=0.001^{*}, \mathrm{p}_{2}=0.883, \mathrm{p}_{3}=0.003^{*} \\
\mathrm{p}_{4}=0.036, \mathrm{p}_{5}=0.495, \mathrm{p}_{6}=0.087\end{array}$} & \\
\hline $\begin{array}{l}\text { Platelets } \\
\left(\text { cells } / \mathrm{mm}^{3}\right)\end{array}$ & & & & & \\
\hline Mean \pm SD & $151525 \pm 50663$ & $146200 \pm 55308$ & $99517 \pm 94156$ & $154382 \pm 87792$ & \multirow[b]{2}{*}{0.176} \\
\hline Range & $67000-310000$ & $59000-310000$ & $102-279000$ & $\begin{array}{l}45000- \\
527000\end{array}$ & \\
\hline
\end{tabular}

SD: standard deviation, WBCs: white blood cells.Hb: hemoglobin, p1: $\mathrm{p}$ value for comparing between Group A and Group B, p2: p value for comparing between Group A and Group CA, p3: p value for comparing between Group A and Group CB, p4: $p$ value for comparing between Group B and Group CA, p5: p value for comparing between Group B and Group CB, p6: p value for comparing between Group CA and Group CB

Concerning the Child-Turcotte-Pugh score classification of group A, group B, group $\mathrm{Ca}$ and group $\mathrm{Cb}$ the number of child,s class "A" patients was 21 (52.50\%), 5 (12.50\%), $1(16.67 \%)$ and 7 $(20.59 \%)$ respectively. The number of child,s class "B" patients was $10(25.5 \%)$, 9 (22.50\%), $2(33.33 \%)$ and $8(23.53 \%)$ respectively, and for the number of child,s class "C" patients was $9(22.50 \%), 26$ (65.0\%), 3 (50.0\%) and 19 (55.88\%) respectively, with statistically significant lower numbers of child,s class " $\mathrm{C}$ " patients $(\mathrm{p}<0.002)$ in the group $A$ compared to other groups, and group B which showed the highest number (Table 3).

Table (3): Comparison between Studied groups regarding Child-Turcotte-Pugh score classification

\begin{tabular}{|c|c|c|c|c|c|c|c|c|c|}
\hline $\begin{array}{l}\text { Groups } \\
\text { Child's class }\end{array}$ & \multicolumn{2}{|c|}{$\begin{array}{c}\text { Group A } \\
(n=40)\end{array}$} & \multicolumn{2}{|c|}{$\begin{array}{c}\text { Group B } \\
(n=40)\end{array}$} & \multicolumn{2}{|c|}{$\begin{array}{c}\text { Group Ca } \\
(n=6)\end{array}$} & \multicolumn{2}{|c|}{$\begin{array}{c}\text { Group Cb } \\
(n=34)\end{array}$} & \multirow[t]{2}{*}{ p-value } \\
\hline & No. & $\%$ & No. & $\%$ & No. & $\%$ & No. & $\%$ & \\
\hline A & 21 & 52.50 & 5 & 12.50 & 1 & 16.67 & 7 & 20.59 & \multirow{3}{*}{$<0.002 *$} \\
\hline B & 10 & 25.00 & 9 & 22.50 & 2 & 33.33 & 8 & 23.53 & \\
\hline $\mathrm{C}$ & 9 & 22.50 & 26 & 65.00 & 3 & 50.00 & 19 & 55.88 & \\
\hline Total & 40 & 100.00 & 40 & 100.00 & 6 & 100.0 & 34 & 100.00 & \\
\hline $\begin{array}{l}\text { Significance } \\
\text { between groups. }\end{array}$ & & & $\begin{array}{l}6.00 \\
4=0.5 \\
\end{array}$ & $\begin{array}{l}{ }^{*}, \mathrm{p}_{2}=0 \\
5, \mathrm{p}_{5}=0\end{array}$ & $\begin{array}{l}210,1 \\
606,\end{array}$ & $\begin{array}{l}3=0.00 \\
36=0.84\end{array}$ & & & \\
\hline
\end{tabular}

$\mathrm{X}$ 2: chi square NS: non-significant, *: significant, $\mathrm{p} 1: \mathrm{p}$ value for comparing between Group A and Group B, p2: $\mathrm{p}$ value for comparing between Group A and Group CA, p3: $\mathrm{p}$ value for comparing between Group A and Group CB, p4: p value for comparing between Group B and Group CA, p5: p value for comparing between Group B and Group CB, p6: p value for comparing between Group CA and Group CB. 
Our study also revealed that regarding the mean values of the splenic size were $13.72 \pm 1.92,17.08 \pm 2.50,16.92 \pm 4.25$ and $17.53 \pm 3.31$ in group $\mathrm{A}$, group $\mathrm{B}$, group $\mathrm{Ca}$ and group $\mathrm{Cb}$ respectively, showing statistically significant difference of $(\mathrm{P}<0.001)$ in the group A which recorded the lowest value compared to group $\mathrm{Cb}$ which recorded the highest value. In the group $\mathrm{A}$, ascites was mild in 11 patients $(27.50 \%)$ and moderate in 6 patients $(15.0 \%)$, while in the group B was mild in 21 patients $(52.50 \%)$ and moderate in 13 patients $(32.50 \%)$, while in the group $\mathrm{Ca}$ was mild in 1 patient $(16.67 \%)$ and was moderate in 1 patient $(16.67 \%)$, In the group $\mathrm{Cb}$, it was mild in 9 patients (26.47\%), moderate in 9 patients $(26.47 \%)$ and marked in 2 patients $(5.88 \%)$, showing statistically significant difference $(\mathrm{p}=0.002)$ in group $\mathrm{Cb}$ which recorded the highest value compared to other groups. Regarding liver size and periportal thickening, no statistically significant difference between the four groups (Table 4).

Table (4): Comparison between Studied groups regarding trans-abdominal ultrasonographic findings

\begin{tabular}{|c|c|c|c|c|c|c|c|c|c|}
\hline $\begin{array}{r}\text { Groups } \\
\text { Parameters }\end{array}$ & \multicolumn{2}{|c|}{$\begin{array}{c}\text { Group A } \\
(n=40)\end{array}$} & \multicolumn{2}{|c|}{$\begin{array}{c}\text { Group B } \\
(n=40)\end{array}$} & \multicolumn{2}{|c|}{$\begin{array}{c}\text { Group Ca } \\
(n=6)\end{array}$} & \multicolumn{2}{|c|}{$\begin{array}{c}\text { Group Cb } \\
(\mathbf{n}=34)\end{array}$} & P-value \\
\hline \multicolumn{10}{|l|}{ Splenic size(cm) } \\
\hline Mean \pm SD & \multicolumn{2}{|c|}{$13.72 \pm 1.92$} & \multicolumn{2}{|c|}{$17.08 \pm 2.50$} & \multicolumn{2}{|c|}{$16.92 \pm 4.25$} & \multicolumn{2}{|c|}{$17.53 \pm 3.31$} & \multirow[b]{2}{*}{$<0.001$} \\
\hline Range & \multicolumn{2}{|c|}{$10.00-17.70$} & \multicolumn{2}{|c|}{$11.50-23.00$} & \multicolumn{2}{|c|}{\begin{tabular}{|c|}
$12.00-$ \\
22.00
\end{tabular}} & \multicolumn{2}{|c|}{$12.00-24.00$} & \\
\hline $\begin{array}{l}\text { Significance } \\
\text { between groups }\end{array}$ & \multicolumn{8}{|c|}{$\begin{array}{c}\mathrm{p}_{1}<0.001^{*}, \mathrm{p}_{2}=0.038^{*}, \mathrm{p}_{3}<0.001^{*}, \\
\mathrm{p}_{4}=0.999, \mathrm{p}_{5}=0.887, \mathrm{p}_{6}=0.955\end{array}$} & \\
\hline & No. & $\%$ & No. & $\%$ & No. & $\%$ & No. & $\%$ & \\
\hline \multicolumn{9}{|l|}{ Ascites } & \multirow{5}{*}{0.002} \\
\hline No & 23 & 57.50 & 6 & 15.00 & 4 & 66.67 & 14 & 41.18 & \\
\hline Mild & 11 & 27.50 & 21 & 52.50 & 1 & 16.67 & 9 & 26.47 & \\
\hline Moderate & 6 & 15.00 & 13 & 32.50 & 1 & 16.67 & 9 & 26.47 & \\
\hline Marked & 0 & 0.00 & 0 & 0.00 & 0 & 0.00 & 2 & 5.88 & \\
\hline Total & 40 & 100.0 & 40 & 100.0 & 6 & 100.0 & 34 & 100.0 & \\
\hline $\begin{array}{l}\text { Significance } \\
\text { between groups }\end{array}$ & \multicolumn{8}{|c|}{$\begin{array}{l}\mathrm{p}_{1}<0.001^{*}, \mathrm{p}_{2}=1.000, \mathrm{p}_{3}=0.235 \\
\mathrm{p}_{4}=0.031^{*}, \mathrm{p}_{5}=0.014^{*}, \mathrm{p}_{6}=0.725\end{array}$} & \\
\hline \multicolumn{10}{|l|}{ Liver size } \\
\hline Average & 30 & 75.00 & 24 & 60.00 & 3 & 50.00 & 20 & 58.82 & \multirow{3}{*}{0.321} \\
\hline Shrunken & 6 & 15.00 & 14 & 35.00 & 3 & 50.00 & 12 & 35.29 & \\
\hline Enlarged & 4 & 10.00 & 2 & 5.00 & 0 & 0.00 & 2 & 5.88 & \\
\hline Total & 40 & 100.0 & 40 & 100.0 & 6 & 100.0 & 34 & 100.0 & \\
\hline \multicolumn{10}{|l|}{$\begin{array}{l}\text { Periportal } \\
\text { thickening: }\end{array}$} \\
\hline No & 34 & 85.00 & 33 & 82.50 & 4 & 66.67 & 27 & 79.41 & \multirow{2}{*}{0.721} \\
\hline Yes & 6 & 15.00 & 7 & 17.50 & 2 & 33.33 & 7 & 20.59 & \\
\hline Total & 40 & 100.0 & 40 & 100.0 & 6 & 100.0 & 34 & 100.0 & \\
\hline
\end{tabular}

$\mathrm{X} 2$ : chi square NS: non-significant, SD: standard deviation, $\mathrm{p} 1: \mathrm{p}$ value for comparing between Group A and Group B, p2: $p$ value for comparing between Group A and Group CA, p3: $p$ value for comparing between Group A and Group CB, p4: p value for comparing between Group B and Group CA, p5: p value for comparing between Group B and Group CB, p6: p value for comparing between Group CA and Group CB. 
The mean values of the splenic vein diameter (SVD) were 9.98 \pm 1.36 , $10.58 \pm 1.71,12.92 \pm .88$ and $11.94 \pm 2.01$ in group $\mathrm{A}$, group $\mathrm{B}$, group $\mathrm{Ca}$ and groupCb respectively, showing statistically significant difference $(\mathrm{p}<0.001)$ between group A which recorded the lowest value compared to group $\mathrm{Ca}$ which recorded the highest value. Splenic vein cross sectional area was found to be $0.80 \pm 0.21$, $0.90 \pm 0.29,1.34 \pm 0.36$ and $1.15 \pm 0.35$ in group $\mathrm{A}$, group $\mathrm{B}$, group $\mathrm{Ca}$ and groupCb respectively, showing statistically significant difference $(\mathrm{p}<0.001)$ between group A which recorded the lowest value compared to group $\mathrm{Ca}$ which recorded the highest value.M. SVV was found to be $13.58 \pm 2.15,12.94 \pm 1.70,11.17 \pm 2.23$ and $12.43 \pm 2.56$ in group $\mathrm{A}$, group $\mathrm{B}$, group $\mathrm{Ca}$ and groupCb respectively, showing statistically significant difference $(p=0.026)$ between group $\mathrm{Ca}$ which recorded the lowest value compared to group A which recorded the highest value. Splenic vein congestion index (S.CI) was found to be $0.06 \pm 0.02, \quad 0.07 \pm 0.02$, $0.12 \pm 0.02$ and $0.09 \pm 0.02$ in group $\mathrm{A}$, group $\mathrm{B}$, group $\mathrm{Ca}$ and groupCb respectively, showing statistically significant difference $(p<0.001)$ between group A which recorded the lowest value compared to group $\mathrm{Ca}$ which recorded the highest value. The direction of flow in splenic vein was found to be normal in all patients in group $\mathrm{A}$, and it was abnormal in 3 patients $(7.5 \%)$ in group B, 2 patients $(33.33 \%)$ in group $\mathrm{Ca}$ and 4 patients $(11.76 \%)$ in group $\mathrm{Cb}$, showing statistically significant difference $(\mathrm{p}=0.019)$ of abnormal blood direction between group A which recorded the lowest value compared to group $\mathrm{Ca}$ which recorded the highest value (Table 5). 
OSAMA S. A. A. GHALWASH et al.,

Table (5): Comparison between Studied groups regarding trans-abdominal doppler ultra-sonographic findings of splenic vein $(\mathrm{SV})$

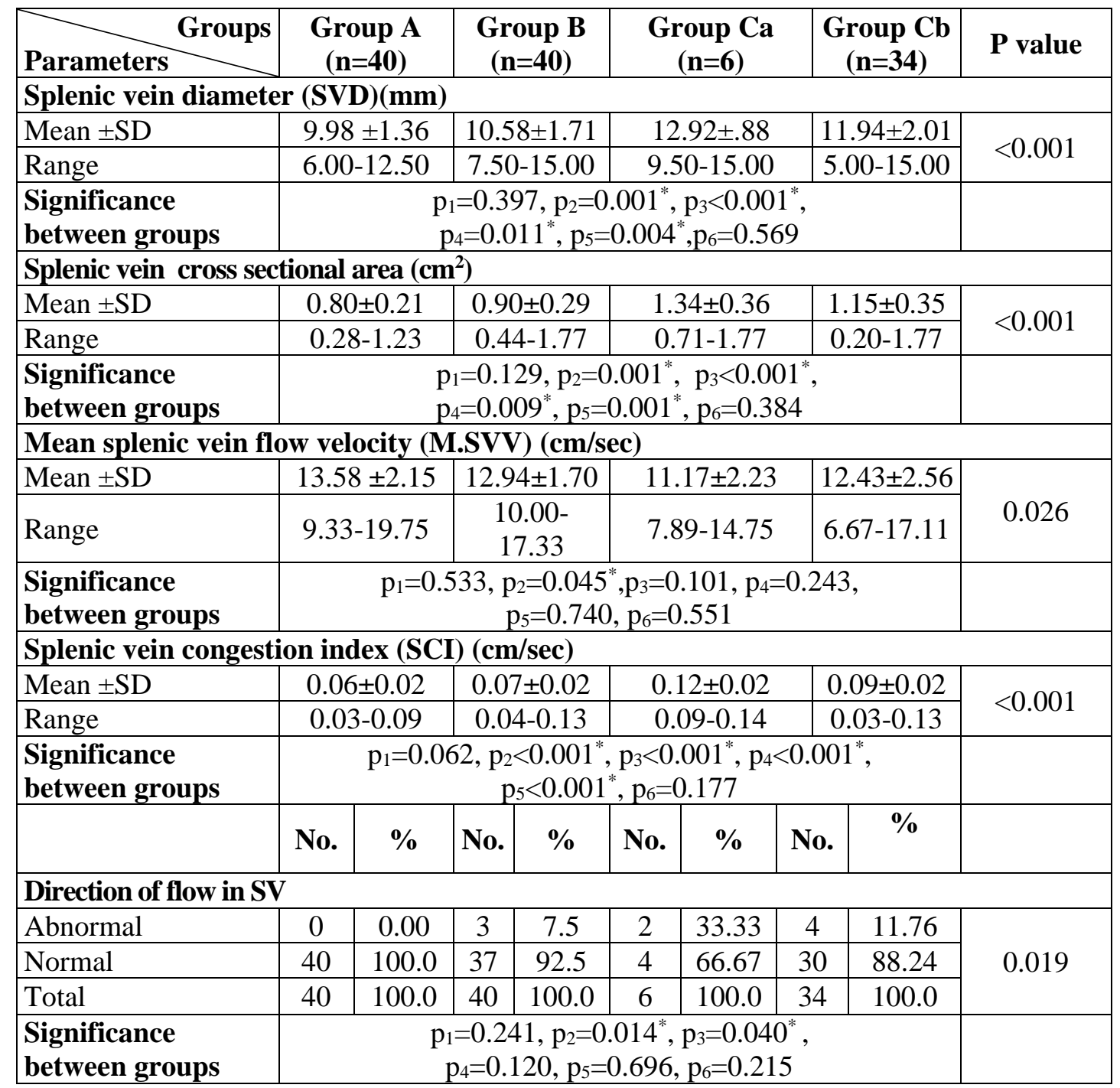

X2: Chi squar, SV: splenic vein.

SD: standard deviation.

p1: $p$ value for comparing between Group A and Group B.

p2: $p$ value for comparing between Group A and Group CA.

p3: $p$ value for comparing between Group A and Group CB.

p4: $p$ value for comparing between Group B and Group CA.

p5: $p$ value for comparing between Group B and Group CB.

p6: $p$ value for comparing between Group CA and Group CB.

Portal vein diameter (PVD) was found to be $13.32 \pm 1.06,15.40 \pm 2.05,14.50 \pm 3.35$ and $15.32 \pm 1.81$ in group A, group B, group $\mathrm{Ca}$ and group $\mathrm{Cb}$ respectively, showing statistically significant difference $(\mathrm{p}<0.001)$ between group A which recorded the lowest value compared to group $\mathrm{Cb}$ which recorded the highest value. Portal vein cross sectional area was found to be $1.40 \pm 0.21,1.89 \pm 0.59$, $1.73 \pm 0.74$ and $1.87 \pm 0.45$ in group $\mathrm{A}$, group $\mathrm{B}$, group $\mathrm{Ca}$ and group $\mathrm{Cb}$ respectively, showing statistically significant difference $(\mathrm{p}<0.001)$ between group A which recorded the lowest value compared to group B which recorded the 
highest value. M. PVV was found to be $11.50 \pm 1.79,13.27 \pm 2.98,8.14 \pm 0.80$ and $9.27 \pm 2.07$ in group A, group B, group Ca and group $\mathrm{Cb}$ respectively, showing statistically significant difference $(\mathrm{p}<0.001)$ between group $\mathrm{Ca}$ which recorded the lowest value compared to group B which recorded the highest value. Portal vein congestion index (P.CI) was found to be $0.12 \pm 0.03,0.15 \pm 0.04$, $0.22 \pm 0.10$ and $0.21 \pm 0.05$ in group $\mathrm{A}$, group $\mathrm{B}$, group $\mathrm{Ca}$ and group $\mathrm{Cb}$ respectively, showing statistically significant difference $(\mathrm{p}<0.001)$ between group A which recorded the lowest value compared to group $\mathrm{Ca}$ which recorded the highest value. Portal vein thrombosis (PVT) not present in group A and group $\mathrm{B}$, while PVT was present in 3 patients (50.0\%) and 7 patients (20.59\%) in group $\mathrm{Ca}$ and group $\mathrm{Cb}$ respectively, showing statistically significant difference $(\mathrm{p}<0.001)$ between group $\mathrm{Ca}$ which recorded the highest value compared to other groups. Direction of blood flow in portal vein was found to be normal in 35 patients $(87.50 \%)$ in group A, $34(85.0 \%)$ patients in group B, 3 patients $(50.0 \%)$ in group $\mathrm{Ca}$, and 24 patients $(70.59 \%)$ in group $\mathrm{Cb}$, showing no statistically significant difference between the studied groups (Table 6). 
Table (6): Comparison between Studied groups regarding trans-abdominal doppler ultra-sonographic findings of portal vein (PV)

\begin{tabular}{|c|c|c|c|c|c|c|c|c|c|}
\hline $\begin{array}{ll}\text { Parameters } & \text { Groups } \\
\end{array}$ & \multicolumn{2}{|c|}{$\begin{array}{c}\text { Group A } \\
(n=40)\end{array}$} & \multicolumn{2}{|c|}{$\begin{array}{c}\text { Group B } \\
(n=40)\end{array}$} & \multicolumn{2}{|c|}{$\begin{array}{c}\text { Group Ca } \\
(n=6)\end{array}$} & \multicolumn{2}{|c|}{$\begin{array}{c}\text { Group Cb } \\
(\mathbf{n}=34)\end{array}$} & $\begin{array}{c}\mathbf{P} \\
\text { value }\end{array}$ \\
\hline $\begin{array}{l}\text { Portal vein diameter (PVD) } \\
(\mathbf{m m}) \\
\text { Mean } \pm \mathrm{SD} \\
\text { Range }\end{array}$ & \multicolumn{2}{|c|}{$\begin{array}{l}13.32 \pm 1.06 \\
10.00- \\
14.50\end{array}$} & \multicolumn{2}{|c|}{$\begin{array}{c}15.40 \pm 2.05 \\
13.30- \\
25.00\end{array}$} & \multicolumn{2}{|c|}{$\begin{array}{c}14.50 \pm 3.35 \\
10.00- \\
18.50\end{array}$} & \multicolumn{2}{|c|}{$\begin{array}{c}15.32 \pm 1.81 \\
12.50- \\
20.00\end{array}$} & $<0.001$ \\
\hline $\begin{array}{l}\text { Significance between } \\
\text { groups }\end{array}$ & \multicolumn{8}{|c|}{$\begin{array}{c}\mathrm{p}_{1}<0.001^{*}, \mathrm{p}_{2}=0.438, \mathrm{p}_{3}<0.001^{*} \\
\mathrm{p}_{4}=0.664, \mathrm{p}_{5}=0.998, \mathrm{p}_{6}=0.727\end{array}$} & \\
\hline $\begin{array}{l}\text { Portal vein cross sectional area } \\
\left(\mathbf{c m}^{2}\right) \\
-\quad \\
-\quad \text { Mean } \pm \text { SD } \\
\end{array}$ & \multicolumn{2}{|c|}{$\begin{array}{l}1.40 \pm 0.21 \\
0.79-1.65\end{array}$} & \multicolumn{2}{|c|}{$\begin{array}{c}1.89 \pm 0.59 \\
1.39-4.91\end{array}$} & \multicolumn{2}{|c|}{$\begin{array}{l}1.73 \pm 0.74 \\
079-269\end{array}$} & \multicolumn{2}{|c|}{$\begin{array}{c}1.87 \pm 0.45 \\
1.23-3.14\end{array}$} & $<0.001$ \\
\hline $\begin{array}{l}\text { Significance between } \\
\text { groups }\end{array}$ & \multicolumn{8}{|c|}{$\begin{array}{c}\mathrm{p}_{1}<0.001^{*}, \mathrm{p}_{2}=0.028^{*}, \mathrm{p}_{3}<0.001^{*} \\
\mathrm{p}_{4}=0.554, \mathrm{p}_{5}=0.862, \mathrm{p}_{6}=0.621\end{array}$} & \\
\hline $\begin{array}{l}\text { Mean portal vein flow } \\
\text { velocity }(\text { M.PVV) }(\mathbf{c m} / \mathbf{s e c}) \\
\text { - } \quad \text { Mean } \pm \text { SD } \\
\text { Range }\end{array}$ & \multicolumn{2}{|c|}{$\begin{array}{l}11.50 \pm 1.79 \\
9.42-16.63\end{array}$} & \multicolumn{2}{|c|}{$\begin{array}{l}13.27 \pm 2.98 \\
9.06-19.70\end{array}$} & \multicolumn{2}{|c|}{$8.14 \pm 0.80$} & \multicolumn{2}{|c|}{$9.27 \pm 2.07$} & $<0.001$ \\
\hline $\begin{array}{l}\text { Significance between } \\
\text { groups }\end{array}$ & \multicolumn{8}{|c|}{$\begin{array}{c}\mathrm{p}_{1}=0.004^{*}, \mathrm{p}_{2}=0.006^{*}, \mathrm{p}_{3}<0.001^{*} \\
\mathrm{p}_{4}<0.001^{*}, \mathrm{p}_{5}<0.001^{*}, \mathrm{p}_{6}=0.687\end{array}$} & \\
\hline $\begin{array}{l}\text { Portal vein congestion index } \\
\text { (P.CI) }(\mathbf{c m} / \mathbf{s e c}) \\
\text { Mean } \pm \text { SD } \\
\text { Range }\end{array}$ & \multicolumn{2}{|c|}{$\begin{array}{l}0.12 \pm 0.03 \\
0.07-0.16\end{array}$} & \multicolumn{2}{|c|}{$\begin{array}{c}0.15 \pm 0.04 \\
0.08-0.27\end{array}$} & \multicolumn{2}{|c|}{$0.22 \pm 0.10$} & \multicolumn{2}{|c|}{$0.21 \pm 0.05$} & $<0.001$ \\
\hline $\begin{array}{l}\text { Significance between } \\
\text { groups }\end{array}$ & \multicolumn{8}{|c|}{$\begin{array}{c}\mathrm{p}_{1}=0.020^{*}, \mathrm{p}_{2}<0.005^{*}, \mathrm{p}_{3}<0.001^{*} \\
\mathrm{p}_{4}=0.099^{*}, \mathrm{p}_{5}<0.001^{*}, \mathrm{p}_{6}=0.422\end{array}$} & \\
\hline & No. & $\%$ & No. & $\%$ & No. & $\%$ & No. & $\%$ & $\begin{array}{c}P \\
\text { value }\end{array}$ \\
\hline $\begin{array}{l}\text { Portal vein thrombosis } \\
\text { (PVT) } \\
\text { No } \\
\text { Yes } \\
\text { Total }\end{array}$ & $\begin{array}{c}40 \\
0 \\
40\end{array}$ & $\begin{array}{c}100.0 \\
0.00 \\
100.0\end{array}$ & $\begin{array}{r}40 \\
0 \\
40\end{array}$ & $\begin{array}{c}100.0 \\
0.00 \\
100.0\end{array}$ & $\begin{array}{l}3 \\
3 \\
6\end{array}$ & $\begin{array}{l}50.00 \\
50.00 \\
100.0\end{array}$ & $\begin{array}{c}27 \\
7 \\
34\end{array}$ & $\begin{array}{l}79.41 \\
20.59 \\
100.0\end{array}$ & \multirow[t]{2}{*}{$<0.001$} \\
\hline $\begin{array}{l}\text { Significance between } \\
\text { groups }\end{array}$ & \multicolumn{8}{|c|}{$\begin{array}{c}\mathrm{p}_{1},{ }^{\mathrm{FE}} \mathrm{p}_{2}=0.001^{*},{ }^{\mathrm{FE}} \mathrm{p}_{3}=0.003^{*} \\
{ }^{\mathrm{FE}} \mathrm{p}_{4}=0.001^{*},{ }^{*} \mathrm{p}_{5}=0.003^{*},{ }^{*} \mathrm{FE}_{6}=0.153\end{array}$} & \\
\hline $\begin{array}{ll}\text { Direction of flow in PV } \\
- & \text { Abnormal } \\
- & \text { Normal } \\
- & \text { Total } \\
\end{array}$ & $\begin{array}{c}5 \\
35 \\
40 \\
\end{array}$ & $\begin{array}{l}12.50 \\
87.50 \\
100.0 \\
\end{array}$ & $\begin{array}{c}6 \\
34 \\
40 \\
\end{array}$ & $\begin{array}{l}15.00 \\
85.00 \\
100.0 \\
\end{array}$ & $\begin{array}{l}3 \\
3 \\
6 \\
\end{array}$ & $\begin{array}{l}50.00 \\
50.00 \\
100.0 \\
\end{array}$ & $\begin{array}{l}10 \\
24 \\
34 \\
\end{array}$ & $\begin{array}{l}29.41 \\
70.59 \\
100.0 \\
\end{array}$ & 0.063 \\
\hline
\end{tabular}

X2: Chi square. PV: portal vein. SD: standard deviation.

p1: $p$ value for comparing between Group A and Group B.

p2: $p$ value for comparing between Group A and Group CA.

p3: $p$ value for comparing between Group A and Group CB.

p4: $p$ value for comparing between Group B and Group CA.

p5: $p$ value for comparing between Group B and Group CB.

p6: $\mathrm{p}$ value for comparing between Group CA and Group CB. 
Regarding collaterals, it was found to be present in only 5 patients $(12.50 \%)$ in group A, 9 patients (22.50\%) in group B, 5 patients $(83.33 \%)$ in group $\mathrm{Ca}$ and 29 patients $(85.3 \%)$ in group $\mathrm{Cb}$, showing statistically significant difference $(\mathrm{P}<0.001)$ between group $\mathrm{A}$ which recorded the lowest value compared to group $\mathrm{Cb}$ which recorded the highest value. Hepatic vein (HV) was found to be attenuated in 9 patients $(22.50 \%)$ in group A, 16 patients $(40 \%)$ in group B, 3 patients $(50.0 \%)$ in group $\mathrm{Ca}$ and 16 patients $(47.06 \%)$ in group $\mathrm{Cb}$, showing no statistically significant difference of attenuated hepatic vein $(p<0.103)$ between the studied groups (Table 7).

Table (7): Comparison between Studied groups regarding hepatic vein (HV) and collaterals

\begin{tabular}{|c|c|c|c|c|c|c|c|c|c|}
\hline \multirow[t]{2}{*}{$\begin{array}{c}\text { Groups } \\
\text { Parameters }\end{array}$} & \multicolumn{2}{|c|}{$\begin{array}{c}\text { Group A } \\
(n=40)\end{array}$} & \multicolumn{2}{|c|}{$\begin{array}{c}\text { Group B } \\
(n=40)\end{array}$} & \multicolumn{2}{|c|}{$\begin{array}{c}\text { Group Ca } \\
(n=6)\end{array}$} & \multicolumn{2}{|c|}{$\begin{array}{c}\text { Group Cb } \\
(\mathbf{n}=\mathbf{3 4})\end{array}$} & \multirow[t]{2}{*}{$P$ value } \\
\hline & No. & $\%$ & No. & $\%$ & No. & $\%$ & No. & $\%$ & \\
\hline \multicolumn{9}{|l|}{$\begin{array}{l}\text { Hepatic vein } \\
\text { (HV) }\end{array}$} & \multirow{3}{*}{0.128} \\
\hline Normal & 31 & 77.50 & 24 & 60.00 & 3 & 50.00 & 18 & 52.94 & \\
\hline Attenuate & 9 & 22.50 & 16 & 40.00 & 3 & 3 & 16 & 47.06 & \\
\hline Total & 40 & 100.00 & 40 & 100.00 & 6 & 100.00 & 34 & 100.00 & \\
\hline \multicolumn{10}{|l|}{ Collaterals } \\
\hline No & 35 & 87.5 & 31 & 77.50 & 1 & 16.67 & 5 & 14.7 & \multirow{2}{*}{$<0.001$} \\
\hline Yes & 5 & 12.50 & 9 & 22.50 & 5 & 83.33 & 29 & 85.3 & \\
\hline Total & 40 & 100.00 & 40 & 100.00 & 6 & 100.00 & 34 & 100.00 & \\
\hline $\begin{array}{l}\text { Significance } \\
\text { between } \\
\text { groups }\end{array}$ & \multicolumn{8}{|c|}{$\begin{array}{l}\mathrm{p}_{1}=0.239, \mathrm{p}_{2}=0.001^{*}, \mathrm{p}_{3}<0.001^{*}, \\
\mathrm{p}_{4}=0.007^{*}, \mathrm{p}_{5}<0.001^{*}, \mathrm{p}_{6}=1.000^{*},\end{array}$} & \\
\hline
\end{tabular}

$\chi 2:$ Chi square.

$\mathrm{p} 1$ : $\mathrm{p}$ value for comparing between Group A and Group B.

p2: $p$ value for comparing between Group A and Group CA.

p3: $p$ value for comparing between Group A and Group CB.

p4: $p$ value for comparing between Group B and Group CA.

p5: $\mathrm{p}$ value for comparing between Group B and Group CB.

p6: $p$ value for comparing between Group CA and Group CB.

\section{DISCUSSION}

In our study, there were no statistically significant differences between the studied groups regarding hemoglobin level, platelet count and INR. These results came in agreement with Sharma et al. (2017) that found that there was no significant difference between esophageal and gastric varices regarding platelets. Also, agreed with the study conducted by Rezayat et al. (2014) who evaluated changes of doppler indices in gastric varices patients with and without gastroesophageal varices.On the other hands, Ali et al. (2015) found that there was a significant decrease in the mean values of platelet count/ spleen diameter ratio in cirrhotic patients with gastric varices in comparison to other patients without gastric varices.

Our study showed statistically significant difference of the mean values of the splenic size in group $\mathrm{Cb}$ which recorded the highest value compared to 
group A which recorded the lowest value. This agreed with the study of Ozdil et al. (2016). On the other hand, Mahassadi et al. (2012) found a lower diagnostic accuracy of splenic size (in Ivorian cirrhotic patients) suggesting that splenomegaly in the African context might not be useful as predictor of gastroesophageal varices.

Ascites in our study showed statistically significant difference where it was marked in group $\mathrm{Cb}$ which recorded the highest value compared to other groups. This finding was in concordance with the results of Ramzy et al. (2015) and Al-Azhary et al. (2018) who found that ascites in the group I (patients with gastroesophageal varices) was more than group II (patients without gastric varices).

In the current study, splenic vein diameter, splenic vein cross sectional area, and splenic congestion index significantly increased among group $\mathrm{Ca}$ and group Cbthan group $\mathrm{A}$ and group $\mathrm{B}$, and this came in concordance with the study of Esmat et al. (2012) who found a statistically significant correlation between the presence and grade of gastroesophageal varices with the splenic diameter, and also was in concordance with results of Rezayat et al. (2014) who found that in half of patients with portal hypertension the splenic vein diameter increases to more than $10 \mathrm{~mm}$. The mean diameter of splenic vein in patients without GVs was 9.4 and was 10.8 in those with GVs, but the difference was not significant.

In our study, portal vein diameter (PVD) significantly increased among group $\mathrm{Cb}$, portal vein congestion index was significantly increased in group $\mathrm{Ca}$.
The mean portal vein flow velocity (M.PVV) significantly decreased among group $\mathrm{Ca}$ and the cross sectional area of portal vein was significantly increased among group B, This results agreed with the study done by Mostafa et al. (2013) who found that the ultra- sonographic parameters showed a significant increase in the splenic diameter and PVD between control group and studied subgroups.On the other hand, the study conducted by Rezayat et al. (2014) reported nosignificant difference between those patients with and without gastroesophageal varices for portal vein diameter. In the study of Chouhan et al. (2015), it was found that there no statistically significant differences in portal vein velocity, congestion index and liver vascular index among the three studied groups.

Regarding portal vein thrombosis (PVT), our study revealed that group $\mathrm{Ca}$ showed statistically significant difference compared to other groups, and this came in concordance with Sharma et al. (2017) who reported that, with portal vein occlusion, both esophageal and gastric varices may develop in the absence of cirrhosis and in this setting varices were most commonly isolated gastric varices rather than gastroesophageal.

Regarding direction of flow in portal vein, our study showed no statistically significant difference between the four groups. This agreed with the study conducted by Rezayat et al. (2014).

Heikal (2020) demonstrated that, cirrhosis is combined with increased intrahepatic resistance which increases pressure in the portal vein (PV) which enhances the opening up of various 
collateral pathways. These hemodynamic events are responsible for the progressive fall in the portal venous blood flow velocity with increasing severity of the portal hypertension.

In our study, presence of collaterals showed statistically significant difference between group $\mathrm{Cb}$ which recorded the highest value compared to group A which recorded the lowest value. This agreed with El-Assaly et al. (2020) who reported that, the commonest type of collaterals draining into superior vena cava is the peri-gastric type, and detected esophageal and paraesophageal collaterals in $70 \%$ of cases and peri-gastric in $76.7 \%$ of cases.

\section{CONCLUSION}

Doppler ultrasound can be an easy, cheap and safe alternative, while platelet count has no any significance in predicting of gastric varices.

\section{REFERENCES}

1. Al-Azhary S, Zaky S, Hussein A andAbd El Fadeel M. (2018): Doppler study of splenic artery and renal artery resistive index as a predictive of esophageal varices and its bleeding risk in Egyptian cirrhotic patients, Al-Azhar Medical Journal, 47(3):587-602.

2. Ali AA, Badawy AM, Sonbol AA andAyad ME. (2015): Study of the relationship between blood ammonia level and esophageal varices in patients with liver cirrhosis. AfroEgyptian Journal of Infectious and Endemic Diseases, 5(2):78-85.

3. Chouhan A, Trikha S, Dhawle S, Nagwanshi $J$ and Chandra S. (2015): A study of correlation of esophageal varices in cirrhotic patients with portal haemodynamics with special reference to portal vein diameter, portal vein velocity, congestion index, liver vascular index. Journal of Evolution of Medical and Dental Sciences,4(1):59-67.

4. DeFranchis $R$ and Faculty BV. (2015): Expanding consensus in portal hypertension:
Report of the Baveno VI Consensus Workshop: Stratifying risk and individualizing care for portal hypertension. J Hepatol., 63(3): 743-752.

5. Di Giorgio A and D'Antiga L. (2019): Portal hypertension. InPediatric Hepatology and Liver Transplantation. Springer, Cham., 25(4): 299-327.

6. El-Assaly H, Metwally LI, Azzam H and Seif-Elnasr MI. (2020): A comparative study of multi-detector CT portography versus endoscopy in evaluation of gastro-esophageal varices in portal hypertension patients. Egyptian Journal of Radiology and Nuclear Medicine. 51(1):5-6.

7. Esmat S., Omarn D and Rashid L. (2012): Can we consider the right hepatic lobe size/albumin ratio a noninvasive predictor of oesophageal varices in hepatitis $\mathrm{C}$ virusrelated liver cirrhotic Egyptian patients. Eur. J. Intern. Med., 23 (3): 67-72.

8. Garcia-Tsao G, Abraldes JG, Berzigotti A and Bosch J. (2017): Portal hypertensive bleeding in cirrhosis: risk stratification, diagnosis, and management: 2016 practice guidance by the American Association for the study of liver diseases. JHepatology,65(1):310-335.

9. Heikal I. (2020): Association between Portal Vein Colored Doppler Ultrasound Findings and Severity of Liver Disease in Cirrhotic Patients with Portal Hypertension. Al-Azhar International Medical Journal, 1(1):232-237.

10. Jesus C.(2017): Portal haemodynamics in cirrhotic patients attending to Arkansas Valley Regional Medical Center. Liver International, 2(3):105-110.

11. Mahassadi A.K., Bathaix F.Y. and Assi C. (2012): Clinical Study: Usefulness of Noninvasive Predictors of Oesophageal Varices in Black African Cirrhotic Patientsin C`ote d'Ivoire (West Africa). Gastroenterology Research and Practice, 19(2):10-11.

12. Mostafa HM, Eid KA, Meguid MM and Mohamed SA. (2013): Comparative Study between Right Liver Lobe Diameter/Albumin Ratio and Platelet Count/Spleen Diameter 
Ratio as a Non-Invasive Predictor of Oesophageal Varices in Patients with Liver Cirrhosis. ResearchGate,81(1):875-885.

13. Ozdil K, Ozturk O, Çalık ES, Akbas ES, Kanat $E$ and Calıskan Z. (2016): Relationship between size of varices and platelet count/spleen size ratio in cirrhotic patients. Northern clinics of Istanbul, 3(1):4647.

14. Ramzy I, Abdel Hafez H, Madani $H$ and Sanad N. (2015): Predictive value of noninvasive blood ammonia level for the presence of oesophageal varices in Egyptian patients with liver cirrhosis. Journal of GHR, 4(5): 1613-1617.

15. Rezayat KA, Ghanaei FM, Alizadeh A, Shafaghi A andJandaghi AB. (2014): Doppler surrogate endoscopy for screening esophageal varices in patients with cirrhosis. Hepatitis monthly, 14(1):1-5.

16. Rye K., Scott R., Gerri M., Lawson A., Austin A.,and Freeman J. (2012): Towards Noninvasive Detection of Oesophageal Varices, International Journal of Hepatology, 7(3):112 -113.
17. Sauerbruch T. and Trebicka J. (2014): Future therapy of portal hypertension in liver cirrhosis a guess. Gastroenterology Research and Practice, 12(7):6-9.

18. Sharma BC, Varakanahalli S, Singh JP and Srivastava S. (2017): Gastric varices in cirrhosis vs. extrahepatic portal venous obstruction and response to endoscopic Nbutyl-2-cyanoacrylate injection. Journal of Clinical and Experimental Hepatology, 7(2):97-101.

19. Wael EA, Saad W, Bleibel1 N, Adenaw CE, Wagner C, Anderson JF, Angle AM, AlOsaimi3 MG, Davies $S$ and Caldwell (2014): Thrombocytopenia in Patients with Gastric Varices and the Effect of Balloonoccluded Retrograde Transvenous Obliteration on the Platelet Count. Journal of Clinical Imaging Science, 4(1):24-29.

20. Zeng XQ, Ma LL, Tseng YJ, Chen J, Cui CX and Luo TC. (2017): Endoscopic cyanoacrylate injection with or without lauromacrogol for gastric varices: a randomized pilot study. Journal of Gastroenterology and Hepatology, 32(3):631638. 


\section{تقيىم عدد الصفائح اللدموية والأشعة التلفزيوزىة باللدوبلر على

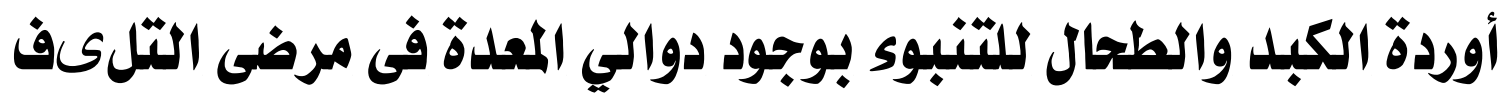 الكبدي}

أسامة سيد احمد أحمد غلوش، جمال محمد محمد سليمان، جلال عبدالحميد أبوفراج، محمد أبو النجا محمد* تمعث

قسمي الكبد والجهاز الهضمي والأمراض المعدية والأثعة التثخيصيةثة، كلية الطب، جامعة الأزهر

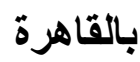

البريد الاليكتروني: gomail.com

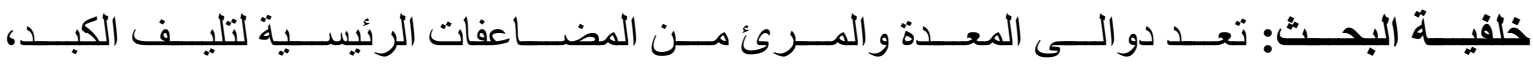

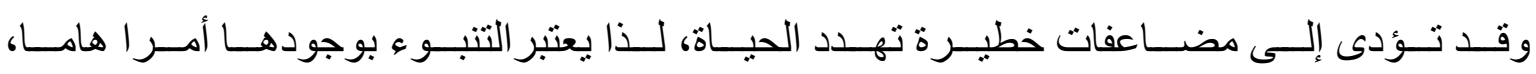

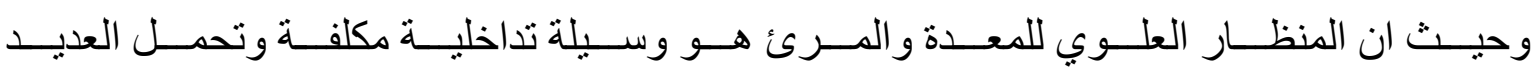

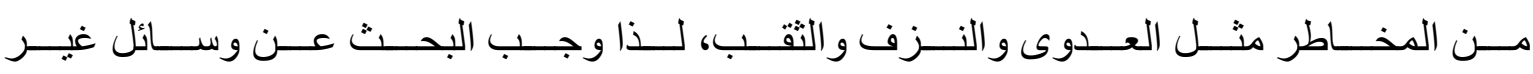
تداخلية للتنبوء بوجود دو الي المعدة.

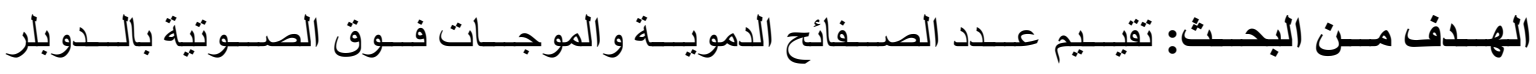

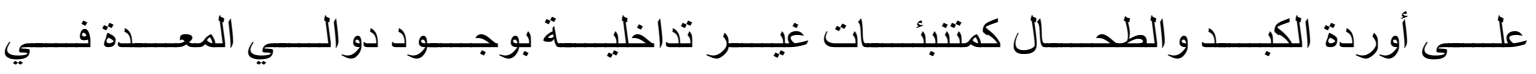

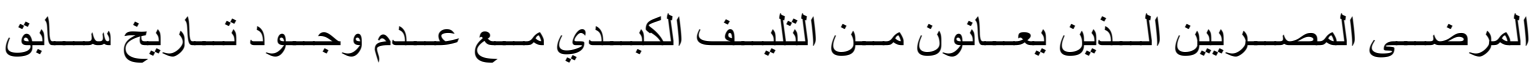
من التنخل بالمنظار أو الجراحة لعلاج إرتفاع ضغط الدم في الوريد البابي.

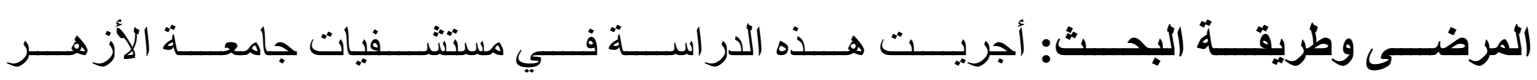

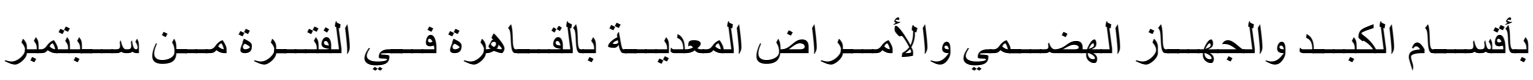

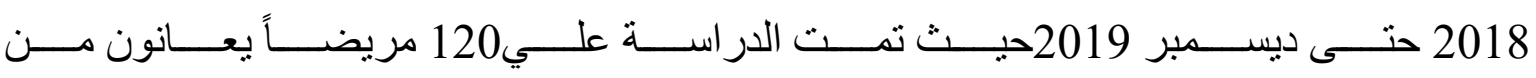

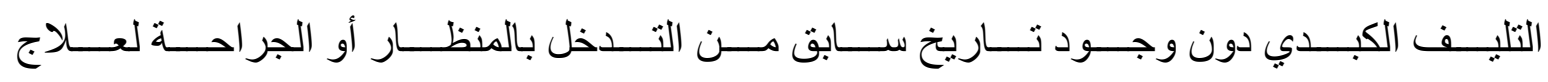

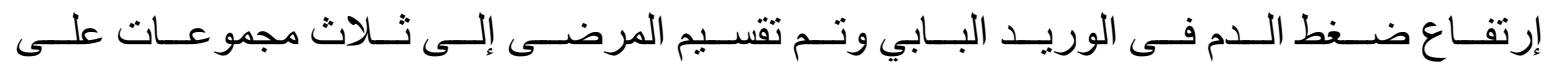

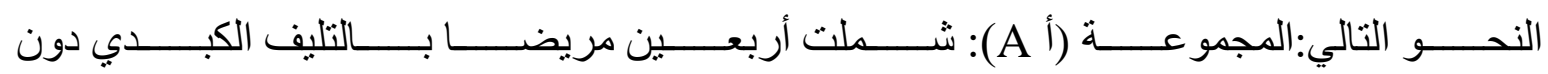

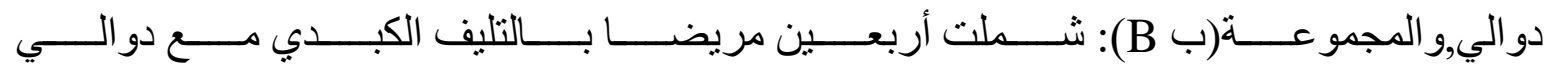

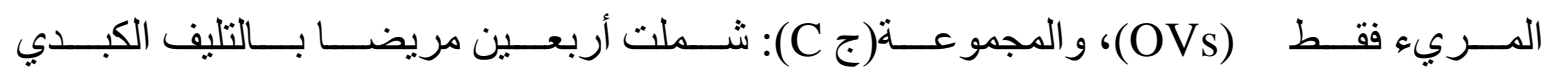

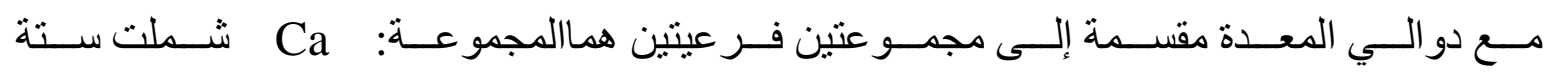




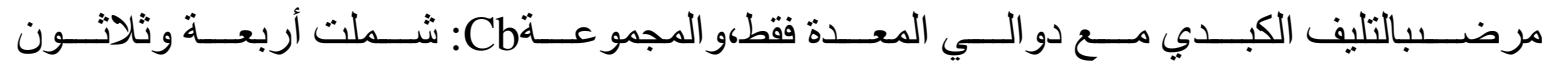
مريضابالتليف الكبدي مع دو الي المعده و المرئ معا.

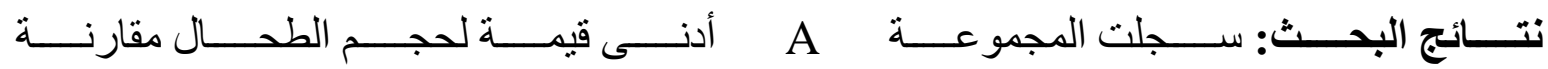

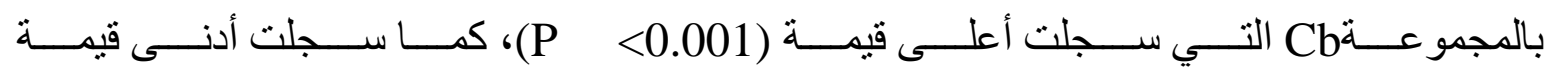

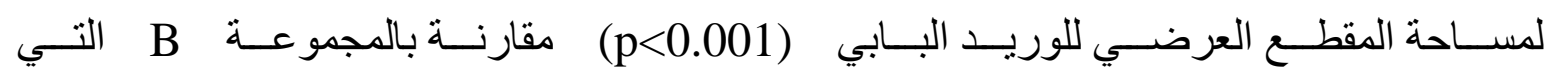

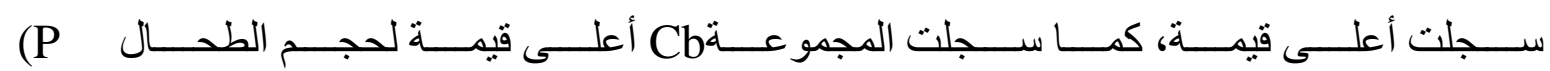

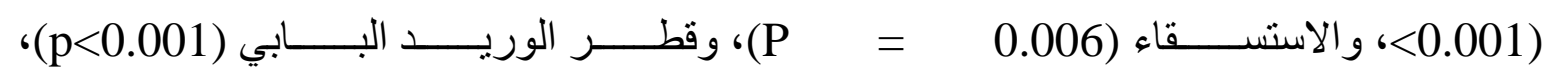

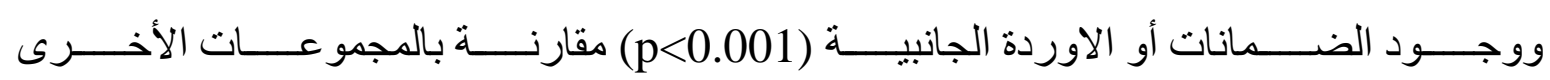

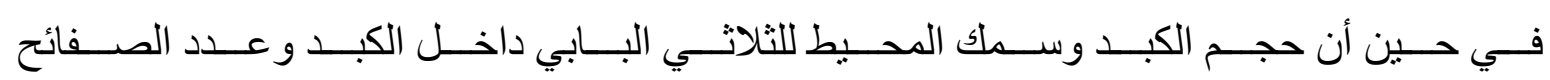

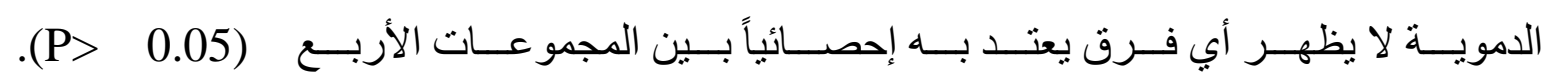

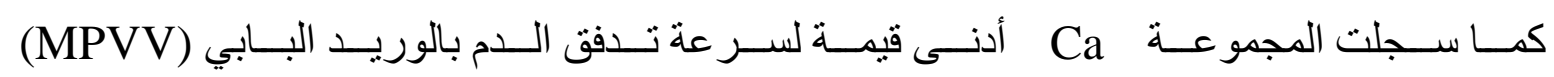

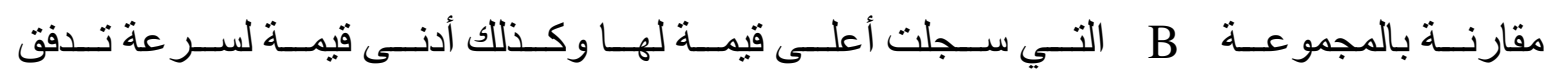

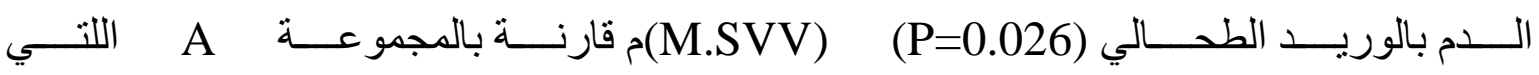

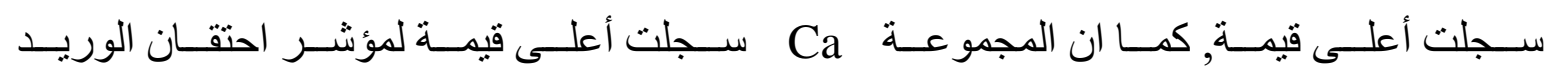
البـابي (P.CI)

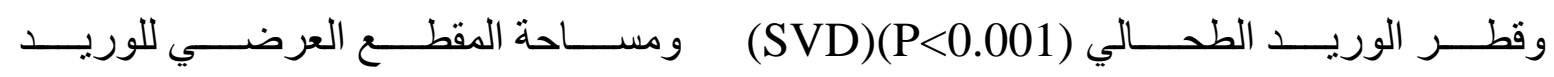

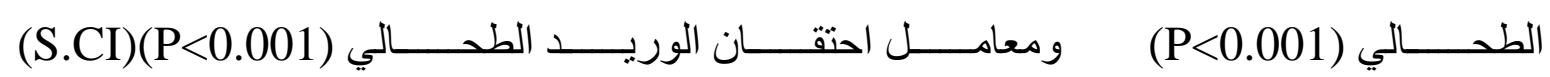

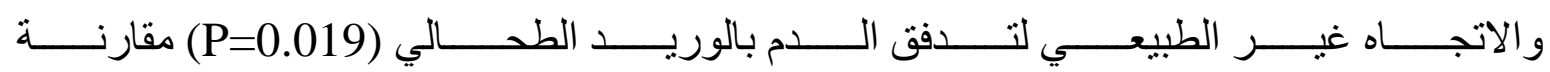

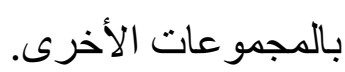

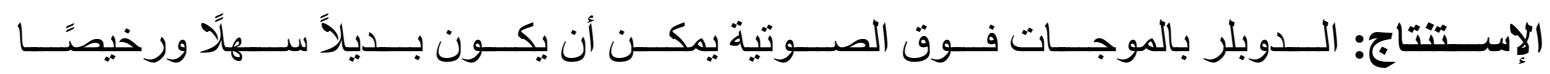

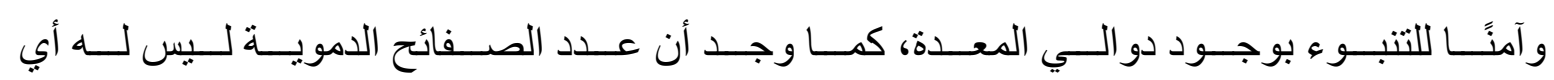
أهمية في التنبوء بدو الي المعدة.

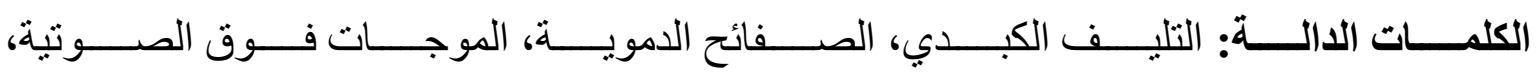
الدوبلر ، الوريد الكبدي، الوريدالطحالي، دو الي المعدة. 